\title{
Still puzzling about a clear definition of pulmonary arterial hypertension in newborns
}

To the Editor:

We read with close attention the report recently published in the European Respiratory Journal by RosenZWeig et al. [1].

We would like to congratulate the authors for their commendable effort, aimed at discussing recent advances, ongoing challenges and specific approaches in the care of children suffering from pulmonary arterial hypertension $(\mathrm{PAH})$, and offer a few comments.

This article is focused on describing the state of the art and future perspectives in paediatric PAH, as formulated by the recent Paediatric Task Force of the 6th World Symposium on Pulmonary Hypertension. They are quite similar to the report of the 5th World Symposium (Nice 2013), in which the unique features of paediatric PAH were highlighted for the first time, but decidedly different from the classification proposed by the Pulmonary Vascular Research Institute Taskforce (Panama 2011) [2, 3].

We agree that the marked age-related differences regarding $\mathrm{PAH}$ aetiology, clinical presentation and outcomes require a different approach in children, especially in infants under the age of 3 months.

In this respect, we would like to add that in persistent PAH of the newborn, the most common cause of transient PAH at that age (involving almost two out of 1000 live and at term births), the criteria for diagnosis are very challenging. These are based on clinical (need for respiratory support because of a $>10 \%$ difference between pre-ductal and post-ductal saturation OR more than two episodes of desaturation $<85 \%$ over a $12 \mathrm{~h}$ period of clinical observation and notwithstanding underlying lung disease optimal medical treatment) as well as echocardiographic features (structurally normal heart; peak gradient of systolic pulmonary arterial pressure $>35 \mathrm{mmHg}$ OR estimated pulmonary arterial pressure greater than two-thirds of the systemic arterial pressure AND presence of right-to-left shunt across a patent foramen ovale and/or patent ductus arteriosus) [4, 5].

Echocardiography is undoubtedly the gold standard for diagnosis, because techniques such as cardiac catheterisation and cardiac magnetic resonance imaging to assess pulmonary vascular resistance, blood flow and myocardial function are currently not feasible in sick newborns [6].

Furthermore, it is the oxygenation index (OI), calculated as

$$
\mathrm{OI}=\text { mean airway pressure } \times \mathrm{FIO}_{2} / \mathrm{PaO}_{2}
$$

where $\mathrm{FIO}_{2}$ is the inspiratory oxygen fraction (\%) and $\mathrm{PaO}_{2}$ is the arterial oxygen tension (in $\mathrm{mmHg}$ ), which is normally used in the paediatric intensive care unit to stratify the severity of hypoxic respiratory failure and prognosis related with persistent PAH. Oxygenation can be continuously and noninvasively monitored by means of pulse oximetry $\left(\mathrm{SpO}_{2}\right)$, and the oxygen saturation index (OSI), calculated as

$$
\mathrm{OSI}=\text { mean airway pressure } \times \mathrm{FIO}_{2} \times 100 / \mathrm{SpO}_{2}
$$

can be another reliable way of assessing the severity of hypoxic respiratory failure [7].

Overall, in our view the above stated refined definition of PAH should be taken into account in newborns under the age of 3 months. We do think that a multidisciplinary team, involving neonatologists,

@ERSpublications

A more in-depth, refined definition of pulmonary arterial hypertension in newborns is needed http://ow.ly/DQH530nDaLz

Cite this article as: Calcaterra G, Fanos V, Bassareo PP. Still puzzling about a clear definition of pulmonary arterial hypertension in newborns. Eur Respir J 2019; 53: 1900005 [https://doi.org/10.1183/ 13993003.00005-2019]. 
paediatricians, cardiologists and respiratory specialists, should be totally committed to the development of specific diagnostic and clinical management strategies in this early life field.

Giuseppe Calcaterra ${ }^{1}$, Vassilios Fanos ${ }^{2}$ and Pier Paolo Bassareo ${ }^{3}$

${ }^{1}$ University of Palermo, Palermo, Italy. ${ }^{2}$ Dept of Surgical Science, Neonatal Intensive Care Unit, Neonatal Pathology Unit, Nursery Unit, University of Cagliari, Cagliari, Italy. ${ }^{3}$ University College of Dublin, Mater Misericordiae University Hospital and Our Lady's Children's Hospital Crumlin, Dublin, Republic of Ireland.

Correspondence: Pier Paolo Bassareo, University College of Dublin, Mater Misericordiae University Teaching Hospital, Eccles St, Inns Quay, Dublin 7, D07 R2WY, Dublin, Republic of Ireland. E-mail: piercard@inwind.it

Received: Jan 032019 | Accepted: Jan 192019

Conflict of interest: None declared.

\section{References}

1 Rosenzweig EB, Abman SH, Adatia I, et al. Paediatric pulmonary arterial hypertension: updates on definition, classification, diagnostics and management. Eur Respir J 2019; 53: 1801916.

2 Galiè N, Simonneau G. The Fifth World Symposium on Pulmonary Hypertension. J Am Coll Cardiol 2013; 62: Suppl. 25, D1-D3.

3 Cerro MJ, Abman S, Diaz G, et al. A consensus approach to the classification of pediatric pulmonary hypertensive vascular disease: report from the PVRI Pediatric Taskforce, Panama 2011. Pulm Circ 2011; 1: 286-298.

4 Clark RH, Kueser TJ, Walker MW, et al. Low-dose nitric oxide therapy for persistent pulmonary hypertension of the newborn. Clinical Inhaled Nitric Oxide Research Group. N Engl J Med 2000; 342: 469-474.

5 Sharma V, Berkelhamer S, Lakshminrusimha S. Persistent pulmonary hypertension of the newborn. Matern Health Neonatol Perinatol 2015; 1: 14.

6 Jain A, Mohamed A, El-Khuffash A, et al. A comprehensive echocardiographic protocol for assessing neonatal right ventricular dimensions and function in the transitional period: normative data and $\mathrm{z}$ scores. J Am Soc Echocardiogr 2014; 27: 1293-1304.

7 Rawat M, Chandrasekharan PK, Williams A, et al. Oxygen saturation index and severity of hypoxic respiratory failure. Neonatology 2015; 107: 161-166.

Copyright @ERS 2019

From the authors:

We appreciate the comments by G. Calcaterra and co-workers regarding the recent summary of the Paediatric Task Force from the 6th World Symposium on Pulmonary Hypertension (WSPH; Nice, 2018) [1]. While many messages endure, including the ongoing emphasis in 2018 on the distinct needs and approach to children with pulmonary hypertension $(\mathrm{PH})$, we have learned much since the last symposium in 2013. Even in the absence of a dramatic change in the therapeutic landscape for children with $\mathrm{PH}$, we examined new and important paediatric data from the past five years that informed our contemporary approach and defined ongoing needs and knowledge gaps for the future.

The Paediatric Task Force further highlighted the importance of focusing on children with WSPH group 3 PH (lung disease/hypoxia). As described in the report, we note the growing awareness of the high rate of $\mathrm{PH}$ in term and preterm infants during the early postnatal period and discuss the important need for better characterisation of incidence, natural history, disease mechanisms, diagnosis, treatment and long-term outcomes in young children and infants [1]. Persistent pulmonary hypertension of the newborn (PPHN) is a clinical syndrome that is defined by the presence of extrapulmonary right-to-left shunt across the foramen ovale or patent ductus arteriosus, which leads to hypoxaemia. There is no specific level of estimated pulmonary artery pressure to define $\mathrm{PH}$ in this setting, but rather, PPHN is defined physiologically by hypoxaemia due to the demonstration of predominant right-to-left shunt, as best defined by echocardiography, and not oxygenation alone. Many neonates, especially after preterm birth, can have evidence of delayed transition of the lung circulation at birth, which can be associated with variable degrees of lung parenchymal disease, lung hypoplasia and other diseases, or may have evidence of 
pulmonary venous hypertension due to left heart dysfunction. Cardiopulmonary interactions during the acute transitional period after birth play key roles in contributing to the degree of hypoxaemia related to shunt and are part of the diagnostic evaluation.

Our definition of PH starts from 3 months of age because the early neonatal period is dynamic with both normal and pathological variations in transition to extrauterine life. By 3 months of age, however, pulmonary haemodynamics are generally similar to those established for other postnatal ages. Again, as discussed in our paper, PPHN is a clinical syndrome with a specific definition of failed circulatory adaptation at birth, but is certainly not synonymous with pulmonary vascular disease. The criteria described by G. Calcaterra and co-workers are not sufficient for $\mathrm{PH}$ [1]. That is, oxygenation index (OI) is not specific for PPHN physiology, nor does it accurately differentiate between the severity of parenchymal lung disease from the degree of shunt as the primary cause of hypoxaemic respiratory failure. As a result, it has no diagnostic role for $\mathrm{PH}$ in sick neonates but is a good marker for the overall severity of cardiopulmonary disease. In other words, many sick neonates, especially with severe respiratory distress syndrome, can have elevated OI but these infants may or may not have PPHN physiology.

As noted by the authors of this letter, there remains a need for developing a better understanding of the different patterns of change in pulmonary artery pressure during the dynamic period after birth in term and preterm infants and related physiological implications. Normal perinatal transition has been previously defined with regard to echocardiogram-derived pulmonary artery pressure over time in the first days of life. Recently, a study of serial changes in estimated levels of pulmonary hypertension describes several different patterns of the transition in preterm infants, demonstrating striking changes in delayed pulmonary vascular transition being associated with degree of prematurity [2]. Of note, those preterm infants with delayed pulmonary vascular transition had the highest risk for late deaths and the subsequent development of bronchopulmonary dysplasia.

In addition, we had previously recommended the use of the category of "developmental lung diseases" under group 3 at the previous WSPH in 2013, which was based on the recognition of high incidence of $\mathrm{PH}$ in preterm infants and term infants, and in association with such diseases as bronchopulmonary dysplasia and congenital diaphragmatic hernia. In 2018, the Paediatric Task Force further noted that this category also includes important but rare disorders of genetic diseases associated with lung and pulmonary vascular disease, including alveolar capillary dysplasia, TBX4 mutations and others [3].

We also strongly advocate the development of a classification system that accurately represents paediatric-specific disease characteristics and helps provide a better approach to paediatric $\mathrm{PH}$. In an effort to enhance the usefulness for children, these proceedings included additional refinements of the WSPH groups, including a separate designation for congenital/acquired cardiovascular conditions leading to post-capillary $\mathrm{PH}$ (group 2.4), developmental lung disorders (group 3.5), other pulmonary artery obstructions (group 4.2) and complex congenital heart disease (group 5.4). Data on the utility and applicability of the PANAMA system as developed with the Pulmonary Vascular Research Institute, however, remain limited [4]. However, the current WSPH Nice system interfaces well with standards established by our adult colleagues [5], and better enables us to "speak the same language" within the broader $\mathrm{PH}$ community, which may be important to enhance transitioning of our patients from the paediatric setting to adult care, as well as sharing clinical care, research and clinical trial experience among the paediatric and adult worlds. Future work is needed to further develop and optimise a classification system suited for paediatric $\mathrm{PH}$ and to demonstrate its clinical and scientific utility.

Erika B. Rosenzweig ${ }^{1}$, Steven H. Abman ${ }^{2}$ and Rolf M.F. Berger ${ }^{3}$

${ }^{1}$ Columbia University Irving Medical Center, New York Presbyterian Hospital, New York, NY, USA. ${ }^{2}$ University of Colorado, Children's Hospital Colorado, Denver, CO, USA. ${ }^{3}$ Center for Congenital Heart Diseases, Dept of Pediatric Cardiology, Beatrix Children's Hospital, University Medical Center Groningen, University of Groningen, Groningen, The Netherlands.

Correspondence: Erika B. Rosenzweig, Division of Pediatric Cardiology, Columbia University Medical Center, New York Presbyterian, 3959 Broadway, CH2N, New York, NY 10032, USA. E-mail: esb14@cumc.columbia.edu

Received: Jan 232019 | Accepted: Jan 232019

Conflict of interest: E.B. Rosenzweig has nothing to disclose. S.H. Abman has nothing to disclose. R.M.F. Berger reports grants and fees for consultancy from Actelion, fees for consultancy Lilly, outside the submitted work.

\section{References}

1 Rosenzweig EB, Abman SH, Adatia I, et al. Paediatric pulmonary arterial hypertension: updates on definition, classification, diagnostics and management. Eur Respir J 2019; 53: 1801916.

2 Mirza H, Garcia JA, Crawford E, et al. Natural history of postnatal cardiopulmonary adaptation in infants born extremely preterm and risk for death or bronchopulmonary dysplasia. J Pediatr 2018; 198: 187-193. 
3 Zhu N, Gonzaga-Jauregui C, Welch CL, et al. Exome sequencing in children with pulmonary arterial hypertension demonstrates differences compared with adults. Circ Genom Precis Med 2018; 11: e001887.

4 Cerro MJ, Abman S, Diaz G, et al. A consensus approach to the classification of pediatric pulmonary hypertensive vascular disease: report from the PVRI Pediatric Taskforce, Panama 2011. Pulm Circ 2011; 1: 286-298.

5 Simonneau G, Montani D, Celermajer DS. Haemodynamic definitions and updated clinical classification of pulmonary hypertension. Eur Respir J 2019; 53: 1801913.

Copyright @ERS 2019 